Pacific Journal of Mathematic 


\title{
CONCERNING SEMI-STRATIFIABLE SPACES
}

\author{
Geoffrey D. Creede
}

In this paper, a class of spaces, called semi-stratifiable spaces is introduced. This class of spaces lies between the class of semi-metric spaces and the class of spaces in which closed sets are $G_{\delta}$. This class of spaces is invariant with respect to taking countable products, closed maps, and closed unions. In a semi-stratifiable space, bicompactness and countable compactness are equivalent properties. A semi-stratifiable space is $F_{\sigma}$-screenable.

A $T_{1}$-space is semi-metric if and only if it is semi-stratifiable and first countable. A completely regular space is a Moore space if and only if it is a semi-stratifiable $p$-space.

The concept of semi-stratifiable spaces as a generalization of semimetric spaces (see Corollary 1.4) is due to E. A. Michael. It appears that all properties of semi-metric spaces which do not depend on first countability also hold in semi-stratifiable spaces. The class of semistratifiable spaces contains all stratifiable spaces [3], all cosmic spaces [13], and all spaces with a $\sigma$-locally finite [15] or $\sigma$-discrete [2] network.

Some of the results of this paper were announced in [5].

Most terms which are not defined in this paper are used as in Kelley [10].

1. Preliminaries.

Definition 1.1. A topological space $X$ is a semi-stratifiable space if, to each open set $U \subset X$, one can assign a sequence $\left\{U_{n}\right\}_{n=1}^{\infty}$ of closed subsets of $X$ such that

(a) $\cup_{n=1}^{\infty} U_{n}=U$,

(b) $U_{n} \subset V_{n}$ whenever $U \subset V$, where $\left\{V_{n}\right\}_{n=1}^{\infty}$ is the sequence assigned to $V$.

A correspondence $U \rightarrow\left\{U_{n}\right\}_{n=1}^{\infty}$ is a semi-stratification for the space $X$ whenever it satisfies conditions (a) and (b) of Definition 1.1.

By comparing the above definition with Definition 1.1 of [3], one can see that, if the correspondence $U \rightarrow\left\{U_{n}\right\}_{n=1}^{\infty}$ is a stratification for $X$, then $U \rightarrow\left\{U_{n}^{\prime}\right\}_{n=1}^{\infty}$, where $U_{n}^{\prime}=\mathrm{Cl} U_{n}$, is a semi-stratification for $X$. In [8], Heath gives an example of a (paracompact) semi-stratifiable space which is not stratifiable.

THEOREM 1.2. A necessary and sufficient condition for $a$ topological space $X$ to be semi-stratifiable is that there be a sequence 
$\left\{g_{i}\right\}_{i=1}^{\infty}$ of functions from $X$ into the collection of open sets of $X$ such that (i) $\bigcap_{i=1}^{\infty} g_{i}(x)=C l\{x\}$ for each $x$, and (ii) if $y$ is a point of $X$ and $\left\{x_{i}\right\}_{i=1}^{\infty}$ is a sequence of points in $X$, with $y \in g_{i}\left(x_{i}\right)$ for all $i$, then $\left\{x_{i}\right\}_{i=1}^{\infty}$ converges to $y$.

Proof. Let $U \rightarrow\left\{U_{n}\right\}_{n=1}^{\infty}$ be a semi-stratification for $X$. For each $i$, define the function $g_{i}$ by $g_{i}(x)=X-(X-\mathrm{Cl}\{x\})_{i}$. The sequence $\left\{g_{i}\right\}_{i=1}^{\infty}$ satisfies conditions (i) and (ii) of the theorem.

Conversely, let $\left\{g_{i}\right\}_{i=1}^{\infty}$ satisfy conditions (i) and (ii) of the theorem. For each $n$ and each open set $U$, let $U_{n}=X-\bigcup\left\{g_{n}(x): x \in X-U\right\}$. Then correspondence $U \rightarrow\left\{U_{n}\right\}_{n=1}^{\infty}$ is a semi-stratification for $X$.

Definition 1.3. A topological space $X$ is semi-metric if there is a distance function $d$ defined on $X$ such that

(1) $d(x, y)=d(y, x) \geqq 0$

(2) $d(x, y)=0$ if and only if $x=y$,

(3) $x$ is a limit point of a set $M$ if and only if $\inf \{d(x, y): y \in M\}=0$. See $[7,11]$.

With the aid of Theorem 3.2 of [7] we have the following relationship between semi-stratifiable spaces and semi-metric spaces.

CoRollary 1.4. A $T_{1}$-space is a semi-metric space if and only if it is a first countable semi-stratifiable space.

\section{Properties of semi-stratifiable spaces.}

THEOREM 2.1. The countable product of semi-stratifiable spaces is semi-stratifiable.

Proof. For each $i$, let $X_{i}$ be a semi-stratifiable space and $\left\{g_{i j}\right\}_{j=1}^{\infty}$ be a sequence of functions on $X_{i}$ satisfying the conditions of Theorem 1.2. Let $X=\prod_{i=1}^{\infty} X_{i}$ and let $\pi_{i}$ be the projection of $X$ onto $X_{i}$. For each $i, j$ and each $x$ in $X$, let $h_{i j}(x)=g_{i j}\left(\pi_{i}(x)\right)$ if $j \leqq i$ and $h_{i j}(x)=$ $X_{i}$ if $j>i$. Now let $g_{j}(x)=\prod_{i=1}^{\infty} h_{i j}(x)$ for each $j$ and $x$. The sequence $\left\{g_{i}\right\}_{i=1}^{\infty}$ satisfies the conditions of Theorem 1.2 and, hence, $X$ is semistratifiable.

THEOREM 2.2. A semi-stratifiable space is hereditarily semistratifiable.

Theorem 2.2 can be proved by taking the natural restriction to the subspace of a semi-stratification of the larger space. In the case of closed subspaces, all semi-stratifications on the subspace can be 
constructed in this manner.

THEOREM 2.3. If $Y$ is a closed subspace of a semi-stratifiable space $X$ and $U \rightarrow\left\{U_{n}\right\}_{n=1}^{\infty}$ is a semi-stratification for $Y$, then there is a semi-stratification $V \rightarrow\left\{V_{n}\right\}_{n=1}^{\infty}$ for $X$ such that $(V \cap Y)_{n}=\left(V_{n} \cap Y\right)$.

Proof. If $W \rightarrow\left\{W_{n}\right\}_{n=1}^{\infty}$ is any semi-stratification for $X$, then let $V_{n}=(V \cap Y)_{n} \cup(V-Y)_{n}$. The correspondence $V \rightarrow\left\{V_{n}\right\}_{n=1}^{\infty}$ is a semistratification for $X$ satisfying $(V \cap Y)_{n}=V_{n} \cap Y$.

By applying Theorem 2.3 with respect to the common subspace, we obtain the following theorem:

THEOREM 2.4. The union of two closed (in the union) semistratifiable spaces is semi-stratifiable.

DEFINITION 2.5. A topological space is $F_{o}$-screenable if every open cover has a $\sigma$-discrete closed refinement which covers the space.

Theorem 2.6 generalizes McAulely's Lemma 1 of [12].

Theorem 2.6. A semi-stratifiable space is $F_{\sigma}$-screenable.

Proof. Let $X$ be a semi-stratifiable space with a semi-stratification $U \rightarrow\left\{U_{n}\right\}_{i=1}^{\infty}$. Let $\left\{O_{\alpha}: \alpha \in I\right\}$ be an open cover of $X$ and let $I$ be wellordered. For each natural number $n$, define: $H_{1 n}=\left(O_{1}\right)_{n}$ and, for each $\alpha>1, H_{a n}=\left(O_{\alpha}\right)_{n}-\cup\left\{O_{\beta}: \beta \in I, \beta<\alpha\right\}$. For each natural number $n$, let $\mathscr{S}_{n}=\left\{H_{\alpha n}: \alpha \in I\right\}$. Then $\mathscr{C}_{n}$ is a discrete collection of closed sets. By the well-ordering on $I, \mathscr{X}=\cup_{n=1}^{\infty} \mathscr{C}_{n}$ covers $X$.

Definition 2.7. A topological space is $\boldsymbol{\aleph}_{1}$-compact if every uncountable subset has a limit point.

THEOREM 2.8. In a semi-stratifiable $T_{1}$-space $X$, the following are equivalent (1) $X$ is Lindelof, (2) $X$ is hereditarily separable, and (3) $X$ is $\boldsymbol{\aleph}_{1}$-compact.

Proof. $\quad(1)=(2)$ Let $X$ be a Lindelöf semi-stratifiable space. Since a Lindelöf space in which open sets are $F_{\sigma}$ is hereditarily Lindelöf, it is sufficient to show that $X$ is separable. Let $\left\{g_{i}\right\}_{i=1}^{\infty}$ be a sequence of functions satisfying the conditions of Theorem 1.2. For each $i$, $\left\{g_{i}(x): x \in X\right\}$ is an open cover of $X$ and, since $X$ is Lindelöf, there is a countable subset $D_{i}$ of $X$ such that $\left\{g_{i}(x): x \in D_{i}\right\}$ is an open cover of $X$. The set $D=\cup_{i=1}^{\infty} D_{i}$ is a countable dense subset of $X$. 
$(2) \Rightarrow(3) \quad$ The proof of this part is well-known.

$(3) \Longrightarrow(1) \quad$ Let $X$ be an $\aleph_{1}$-compact semi-stratifiable $T_{1}$-space. Let $\mathscr{G}$ be an open cover of $X$ and suppose that $\mathscr{G}$ has no countable subcover. By Theorem 2.6, $\mathscr{C}$ has a closed refinement $\mathscr{H}=\cup_{n=1}^{\infty} \mathscr{H}_{n}$ where each $\mathscr{C}_{n}$ is discrete. Since $\mathscr{C}$ has no countable subcover, there is an $n$ such that $\mathscr{H}_{n}$ is uncountable. Let $X^{\prime}$ be a subset of $X$ consisting of exactly one point of each nonempty element of $\mathscr{H}_{n}$. The set $X^{\prime}$ is uncountable and has no limit point.

Theorem 2.8 cannot be strengthened by replacing hereditarily separable by separable. The example of a Moore space which is not metrizable due to R. L. Moore (see [9]) is an example of a separable semi-stratifiable space which is not Lindelöf.

Since, in a Lindelöf space, bicompact is equivalent to countably compact, Theorem 2.8 has the following corollary:

COROLlary 2.9. In a semi-stratifiable $T_{1}$-space, bicompact is equivalent to countably compact.

3. Mappings. It is a well-known theorem [17] that the closed compact image of a separable metric space is a separable metric space. However, there are closed images of separable metric spaces which are not first countable. The following theorem gives a property of metric spaces which is preserved by closed maps.

THEOREM 3.1. The closed image of a semi-stratifiable space is semi-stratifiable.

Proof. Let $f$ be a closed continuous function from a semi-stratifiable space $X$ onto a topological space $Y$. Let $U \rightarrow\left\{U_{n}\right\}_{n=1}^{\infty}$ be a semistratification for $X$. For each open set $V$ of $Y$ and each natural number $n$, let $V_{n}=f\left(\left(f^{-1}[V]\right)_{n}\right)$. The correspondence $V \rightarrow\left\{V_{n}\right\}_{n=1}^{\infty}$ is a semi-stratification for $Y$.

Theorem 3.1 does not remain true if closed is replaced by open.

Theorem 3.1 and Corollary 1.4 imply that the closed image of a semi-metric space is semi-stratifiable. However, it can be shown that the subspace of $\beta N$ (the Stone-Cech compactification of the natural numbers) consisting of $N$ together with one point of $\beta N-N$ is a semistratifiable space which cannot be the closed image of a semi-metric space. It is an open question whether the spaces which are closed images of semi-metric spaces are precisely the semi-stratifiable FréchetUrysohn spaces [2].

4. Moore spaces. In this section, we wish to give necessary and sufficient conditions for a semi-stratifiable space to be a Moore space. 
DEFINITION 4.1. A sequence $\left\{\mathscr{G}_{n}\right\}_{n=1}^{\infty}$ of open covers of a topological space $X$ is a development for $X$ if (1) $\mathscr{G}_{i+1}$ is a refinement for $\mathscr{G}_{i}$ and (2), if $x$ is a point of $X$ and $U$ is an open set in $X$ containing $x$, then there is a natural number $k$ such that $\operatorname{St}\left(x, \mathscr{G}_{k}\right) \subset U$. A Moore space is a regular $T_{1}$-space which has a development. See $[7,14]$.

A Moore space is semi-metric and, hence, is semi-stratifiable. The following example, due to McAuley [11], shows that this implication cannot be reversed.

EXAMPle 4.2. Let $X$ be the $x$-axis of the Cartesian plane $E^{2}$. Let $d$ denote the usual distance function in $E^{2}$ and, if $p \neq q$, let $\alpha(p, q)$ denote the nonobtuse angle (in radians) formed by $X$ and the line through $p$ and $q$. Define a distance function $D$ on $E^{2}$ as follows: $D(p, p)=0$ and, if $p \neq q, D(p, q)=d(p, q)+\alpha(p, q)$. A basis for the topology on $E^{2}$ is $\left\{U_{\varepsilon}(p): p \in E^{2}, \varepsilon>0\right\}$ where $U_{\varepsilon}(p)=\{q: D(p, q)<\varepsilon\}$. Let $S$ denote $E^{2}$ with this topology. If $S$ were a Moore space, it would be second countable, since it is Lindelöf. But $S$ is not second countable since any basis contains uncountably many elements.

Definition 4.3. A $T_{1}$-space $X$ is said to be quasi-complete provided that there is a sequence $\left\{\Re_{n}\right\}_{n=1}^{\infty}$ of open covers of $X$ with the following property: if $\left\{A_{n}\right\}_{n=1}^{\infty}$ is a decreasing sequence of nonempty closed subsets of $X$ and if there exists an element $x_{0} \in X$ such that, for each $n$, there is a $\mathscr{B}_{n} \in \beta_{n}$ with $A_{n} \cup\left\{x_{0}\right\} \subset B_{n}$, then $\bigcap_{n=1}^{\infty} A_{n} \neq \varnothing$.

Definition 4.4 (Borges [4]). A $T_{1}$-space $X$ is a $w \Delta$-space if there exists a sequence $\left\{{\Re_{n}}_{n}\right\}_{n=1}^{\infty}$ of open covers of $X$ such that, if $\left\{A_{n}\right\}_{n=1}^{\infty}$ is a decreasing sequence of nonempty closed subsets of $X$ and there exists $x_{0} \in X$ for which $A_{n} \subset \operatorname{St}\left(x_{0}, \mathscr{B}_{n}\right)$ for all $n$, then $\bigcap_{n=1}^{\infty} A_{n} \neq \varnothing$.

Definition 4.3 is at least formally weaker then Definition 4.4. It is an open question whether all quasi-complete spaces are $w \Delta$-spaces.

Theorem 4.5, due to Heath [7], gives a sufficient condition for a space to be a Moore space.

THEOREM 4.5. A regular $T_{1}$-space $X$ is a Moore space provided that there is a sequence $\left\{g_{i}\right\}_{2=1}^{\infty}$ of functions from $X$ into the topology on $X$ with the following properties: (A) For each $x$ in $X,\left\{g_{i}(x)\right\}_{i=1}^{\infty}$ is a decreasing local base at $x$. (B) If $y$ is a point of $X$ and $\left\{x_{i}\right\}_{i=1}^{\infty}$ is a sequence in $X$ with $y \in g_{i}\left(x_{i}\right)$ for each $i$, then $\left\{x_{i}\right\}_{i=1}^{\infty}$ converges to $y$. (C) If $y$ is a point of $X, U$ is an open subset of $X$ containing $y$, and $\left\{x_{i}\right\}_{i=1}^{\infty}$ is a sequence in $X$ such that, for each $n, y \in g_{n}\left(x_{n}\right)$ and there 
is a natural number $k$ with $\mathrm{Cl}\left[g_{n+k}\left(x_{n+k}\right)\right] \subset g_{n}\left(x_{n}\right)$, then there is a natural number $m$ with $g_{m}\left(x_{m}\right) \subset U$.

THeorem 4.6. A regular $T_{1}$-space is a Moore space if it is a quasi-complete semi-stratifiable space.

Proof. Let $X$ be a regular quasi-complete semi-stratifiable $T_{1}$ space. Let $\left\{\mathscr{B}_{n}\right\}_{n=1}^{\infty}$ be a sequence satisfying the conditions of Definition 4.3 and let $\left\{h_{n}\right\}_{n=1}^{\infty}$ be a sequence satisfying the conditions of Theorem 1.2. For each $x$ in $X$, let $B_{n}(x)$ be a member of $\mathscr{B}_{n}$ containing $x$. For each $x$, let $g_{1}(x)$ be an open subset of $X$ containing $x$ such that Cl $g_{1}(x) \subset B_{1}(x) \cap h_{1}(x)$ and let $g_{n+1}(x)$ be an open subset of $X$ containing $x$ such that $\mathrm{Cl} g_{n+1}(x) \subset B_{n+1}(x) \cap h_{n+1}(x) \cap g_{n}(x)$. The sequence $\left\{g_{n}\right\}_{n=1}^{\infty}$ satisfies the conditions of Theorem 4.5 and, hence, $X$ is a Moore space.

By Proposition 2.8 of [4], we have the following corollary:

COROLlary 4.7. If $X$ is a regular $T_{1}$-space, then the following are equivalent:

(1) $X$ is a Moore space.

(2) $X$ is a semi-stratifiable $w \Delta$-space.

(3) $X$ is a semi-stratifiable quasi-complete space.

If $X$ is a completely regular $T_{1}$-space, let $\beta X$ denote its StoneČech compactfication. The following definition is due to Arhangel'skii $[1,2]$.

Definition 4.8. A completely regular $T_{1}$-space $X$ is a $p$-space provided that there is a sequence $\left\{\mathscr{B}_{n}\right\}_{n=1}^{\infty}$ of collections of open subsets of $\beta X$ such that each $\mathscr{B}_{n}$ covers $X$ and $\bigcap_{n=1}^{\infty}$ St $\left(x, \mathscr{B}_{n}\right) \subset X$ for each point $x$ in $X$.

Lemma 4.9. A p-space is quasi-complete.

Proof. Let $X$ be a $p$-space and let $\left\{\mathscr{B}_{n}\right\}_{n=1}^{\infty}$ satisfy the conditions of Definition 4.8. For each $n$, let $\mathscr{B}_{n}^{\prime}$ be an open cover of $X$ such that, if $B \in \mathscr{B}_{n}^{\prime}$, then $\mathrm{Cl}_{\beta} B$ is contained in some member of $\mathscr{B}_{n}$. Let $\left\{A_{n}\right\}_{n=1}^{\infty}$ be a decreasing sequence of closed subsets of $X$ and $x$ be a point of $X$ such that there is a $B_{n} \in \mathscr{B}_{n}^{\prime}$ with $A_{n} \cup\{x\} \subset B_{n}$ for each

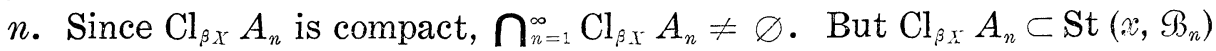
and $\bigcap_{n=1}^{\infty} \mathrm{Cl}_{\beta X} A_{n} \subset X$. Thus, $\bigcap_{n=1}^{\infty} A_{n}=\bigcap_{n=1}^{\infty} \mathrm{Cl}_{\beta X} A_{n}$. Hence, $X$ is quasi-complete. 
It can be seen that, in completely regular spaces, the concepts of $w \Delta$-spaces, $p$-spaces, and quasi-complete spaces are related. The exact relationship between these three concepts is an open problem.

THEOREM 4.10. A completely regular $T_{1}$-space is a Moore space if and only if it is a semi-stratifiable p-space.

Proof. Lemma 4.9 and Theorem 4.6 show that a semi-stratifiable $p$-space is a Moore space.

Conversely, let $X$ be a completely regular Moore space and let $\left\{\mathscr{G}_{n}\right\}_{n=1}^{\infty}$ be a development for $X$. By the remark following Definition 4.1, $X$ is semi-stratifiable. For each $n$, let

$$
\mathscr{B}_{n}=\left\{\beta X-\mathrm{Cl}_{\beta X}(X-G): G \in \mathscr{C}_{n}\right\} .
$$

The sequence $\left\{\mathscr{B}_{n}\right\}_{n=1}^{\infty}$ satisfies the conditions of Definition 4.8. Since $G \subset \beta X-\mathrm{Cl}_{\beta X}(X-G), \mathscr{B}_{n}$ covers $X$. If $x \in X$ and $y \in \beta X-X$, let $U$ and $V$ be disjoint open subsets of $\beta X$ containing $x$ and $y$, respectively. There is a $k$ where $\operatorname{St}\left(x, \mathscr{G}_{k}\right) \subset U \cap X$. Then $y \notin \operatorname{St}\left(x, \mathscr{B}_{k}\right)$. Hence, $X$ is a $p$-space.

Since a locally compact Hausdorff space is a $p$-space, we have the following corollary:

COROLLARY 4.11. A locally compact semi-stratifiable Hausdorff space is a Moore space.

In Theorem 4.10, the condition of complete regularity can be replaced with regularity by using the Wallman compactification $[6,16]$ instead of the Stone-Čech compactification. Appropriate changes will also have to be made in Definition 4.8.

\section{REFERENCES}

1. A. V. Arhangel'skii, On a class of spaces containing all metric and all locally bicompact spaces, Soviet Math. Dokl. 4 (1963), 1051-1055.

2. ——, Mappings and spaces, Russian Math. Surveys 21 (1966), 115-162.

3. C. J. R. Borges, On stratifiable spaces, Pacific J. Math. 17 (1966), 1-16.

4. - On metrizability of topological spaces, Canad. J. Math. 20 (1968), 795-804.

5. G. D. Creede, Semi-stratifiable spaces, Topology Conference, Arizona State University, 1967, Tempe (1968), 318-323.

6. R. M. Brooks, On Wallman compactifications, Fund. Math. 60 (1967), 157-173.

7. R. W. Heath, Arc-wise connectedness in semi-metric spaces, Pacific J. Math. 12 (1962), 1301-1319.

8. — A paracompact semi-metric space which is not an $M_{3}$-space, Proc. Amer. Math. Soc. 17 (1966), 868-870.

9. F. B. Jones, Metrization, Amer. Math. Monthly 73 (1966), 571-576. 
10. J. L. Kelley, General topology, Van Nostrand, Princeton, 1955.

11. L. F. McAuley, A relation between perfect separability, completeness, and normality in semi-metric spaces, Pacific J. Math. 6 (1956), 315-326.

12. - A note on complete collectionwise normality and paracompactness, Proc. Amer. Math. Soc. 9 (1958), 795-799.

13. E. A. Michael, $\aleph_{0}$-spaces, J. Math. Mech. 15 (1966), 983-1002.

14. R. L. Moore, Foundations of point set theory, Amer. Math. Soc. Colloquium Publications, Vol. 13, Amer. Math. Soc., Providence, 1932 (Revised 1962).

15. Akihiro Okuyama, $\sigma$-spaces and closed mappings. I, Proc. Japan Acad. 44 (1968), 472-477.

16. Henry Wallman, Lattices and topological spaces, Ann. of Math. 39 (1938), 112-126. 17. G. T. Whyburn, Analytic topology, Amer. Math. Soc. Colloquium Publications, Vol. 28, Amer. Math. Soc., Providence, 1942.

Received March 17, 1969. This paper is a part of the author's doctoral dissertation written under the direction of $R$. W. Heath. The research was supported by a NASA Traineeship and a NSF Fellowship.

Arizona State University and

Louisiana State UnIVERsity 


\section{PACIFIC JOURNAL OF MATHEMATICS}

\section{EDITORS}

\author{
H. SAMELSON \\ Stanford University \\ Stanford, California 94305 \\ Richard Pierce \\ University of Washington \\ Seattle, Washington 98105
}

J. DUGUNDJI

Department of Mathematics

University of Southern California

Los Angeles, California 90007

BASIL GORDON*

University of California

Los Angeles, California 90024

\section{ASSOCIATE EDITORS}
E. F. BECKENBACH
B. H. NeUmanN
F. WOLF
K. YoSHIDA

\section{SUPPORTING INSTITUTIONS}

UNIVERSITY OF BRITISH COLUMBIA CALIFORNIA INSTITUTE OF TECHNOLOGY

UNIVERSITY OF CALIFORNIA MONTANA STATE UNIVERSITY

UNIVERSITY OF NEVADA

NEW MEXICO STATE UNIVERSITY

OREGON STATE UNIVERSITY

UNIVERSITY OF OREGON

OSAKA UNIVERSITY

UNIVERSITY OF SOUTHERN CALIFORNIA
STANFORD UNIVERSITY

UNIVERSITY OF TOKYO

UNIVERSITY OF UTAH

WASHINGTON STATE UNIVERSITY

UNIVERSITY OF WASHINGTON

AMERICAN MATHEMATICAL SOCIETY CHEVRON RESEARCH CORPORATION TRW SYSTEMS

NAVAL WEAPONS CENTER 


\section{Pacific Journal of Mathematics}

\section{Vol. 32, No. $1 \quad$ January, 1970}

Robert Alexander Adams, Compact Sobolev imbeddings for unbounded domains ........................................ 1

Bernhard Amberg, Groups with maximum conditions .................. 9

Tom M. (Mike) Apostol, Möbius functions of order k............... 21

Stefan Bergman, On an initial value problem in the theory of two-dimensional transonic flow patterns ................... 29

Geoffrey David Downs Creede, Concerning semi-stratifiable spaces ...... 47

Edmond Dale Dixon, Matric polynomials which are higher

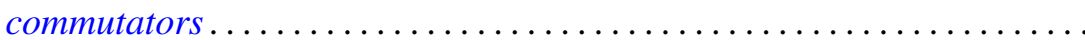

R. L. Duncan, Some continuity properties of the Schnirelmann density.

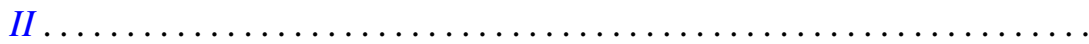

Peter Larkin Duren and Allen Lowell Shields, Coefficient multipliers of $H^{p}$

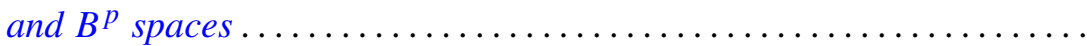

Hector O. Fattorini, On a class of differential equations for vector-valued

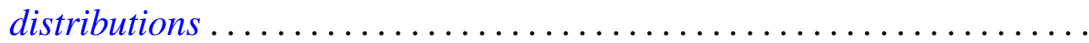

Charles Hallahan, Stability theorems for Lie algebras of derivations. . . . . . 105

Heinz Helfenstein, Local isometries of flat tori ................ 113

Gerald J. Janusz, Some remarks on Clifford's theorem and the Schur

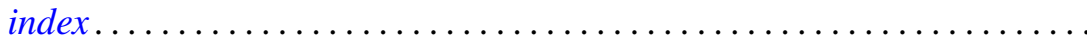

Joe W. Jenkins, Symmetry and nonsymmetry in the group algebras of discrete groups. ...

Herbert Frederick Kreimer, Jr., Outer Galois theory for separable

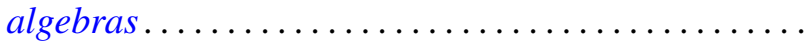

D. G. Larman and P. Mani, On visual hulls

R. Robert Laxton, On groups of linear recurrences. II. Elements of finite order.

Dong Hoon Lee, The adjoint group of Lie groups ...

James B. Lucke, Commutativity in locally compact rings

Charles Harris Scanlon, Rings of functions with certain Lipschitz

$$
\text { properties ............................... }
$$

Binyamin Schwarz, Totally positive differential systems .

James McLean Sloss, The bending of space curves into piecewise helical curves.

James D. Stafney, Analytic interpolation of certain multiplier spaces ...

Patrick Noble Stewart, Semi-simple radical classes.......

Hiroyuki Tachikawa, On left $\mathrm{QF}-3$ rings ...................... 255

Glenn Francis Webb, Product integral representation of time dependent nonlinear evolution equations in Banach spaces.... . . . 